\title{
Suicide risk assessment in elderly individuals
}

\author{
Rui Qi $\underline{T a n}^{1}$, MRCPsych, Chau Sian $\underline{\operatorname{Lim}}^{1}$, MMed, FAMS, Hatta Santoso Ong ${ }^{1}$, MRCPsych
}

\section{INTRODUCTION}

Suicide among elderly individuals is a common and concerning presentation in psychiatry. A local study reported a suicide rate of 31.3 per 100,000 per year among elderly individuals, with elderly Chinese men having the highest rate at 40.3 per 100,000 per year. ${ }^{(1)}$ Although the number of suicides among individuals in other age groups in Singapore has dipped in recent years, the number of suicides among elderly individuals has continued to rise, reaching a highest record of 129 suicides in 2017. ${ }^{(2)}$ This trend is particularly worrying and is expected to continue in view of the growing ageing population in Singapore.

Studies show that over half of the patients who attempted suicide had visited a primary care physician within the four weeks preceding their suicide attempt. ${ }^{(3)}$ This highlights the crucial role of primary care physicians in the early detection of suicidal elderly patients and in providing appropriate intervention. It also calls for an imminent need to better understand the struggles of this unique and vulnerable population.

A proper suicide risk assessment can help ascertain the level of suicide risk and guide its management accordingly. A delicate blend of knowing what questions to ask (i.e. hard skills) and how to ask them (i.e. soft skills) is required to successfully elicit the relevant information.

\section{COMMON STRUGGLES IN AGEING}

Old age is a period associated with losses in many forms. A culmination of chronic physical illnesses and worsening sensory impairment could considerably affect the quality of life of an individual. Deteriorating cognition often triggers anxiety in affected individuals owing to the increasing difficulties that they face while coping independently. A loss of role may also bring about a sense of loss of self. ${ }^{(4)}$ In addition, financial issues may be more prevalent as elderly individuals face rising healthcare expenses and have limited income. Despite these difficulties, many seniors avoid confiding in their family members or friends for fear of burdening them. Coupled with the common misconception that suicidal thoughts are a natural and normal part of ageing, many seniors resist seeking professional help.

\section{UNDERSTANDING THE SPECTRUM OF SUICIDALITY}

Suicidal ideation ranges in severity from having a vague wish of dying to having a specific plan and intent to do so. A person with passive suicidal ideation has thoughts of ending his or her life but has neither plans nor intent to act on them. Conversely, a person who has active suicidal ideation has made such plans and intends to execute them. Understandably, the latter is at a much higher risk of suicide.

A suicide attempt is broadly defined as an act of non-fatal selfinjury performed with an intent to die as a result of the behaviour. There are two important nuances to this definition. Firstly, the act may not necessarily involve actual injury. Secondly, the intent to die can be inferred. Take the example of an elderly man who tried to hang himself from the ceiling with a noose but did not succeed in doing so, as the noose snapped midway. There was no actual injury and the man denied having suicidal ideation during the act. However, it would still be considered a suicide attempt, as it was a self-injurious act and there is no other reasonable explanation regarding why he would try to hang himself.

Suicide attempts should be distinguished from deliberate selfharm, which is a self-injurious act done without suicidal intention. The primary intention in such acts is to relieve unbearable emotions, sensations of unreality or feelings of numbness. An example would be a woman who used a razor blade to cut her wrist to relieve intense emotional distress, which was triggered by a quarrel with her daughter.

\section{RISK FACTORS FOR SUICIDE IN ELDERLY INDIVIDUALS}

Multiple risk factors for suicide among elderly individuals have been identified and summarised in Box $1 .{ }^{(5-8)}$ Some suicide risk factors are modifiable, whereas others are not. Among the various risk factors, a history of suicide attempts, social isolation and the presence of major depressive disorder have been identified as strong predictors of suicide. ${ }^{(6)}$ Elderly patients who experience chronic pain are also at higher risk. ${ }^{(7)}$ Chronic medical illnesses such as cancer, neurological disorders, chronic obstructive pulmonary disease and genitourinary conditions also have a cumulative effect on suicide risk. ${ }^{\left({ }^{(8)}\right.}$

In addition to reducing risk factors, strengthening protective factors can potentially help to mitigate suicide risk. These include strong interpersonal relationships, effective coping strategies, help-seeking behaviour, religious faith and a healthy lifestyle..$^{(9,10)}$

\section{CONDUCTING A SUICIDE RISK ASSESSMENT}

Although conducting a suicide risk assessment might be challenging in a busy local healthcare setting, it is prudent to set aside adequate time to do so. This will enable the clinician to properly practise both the soft and hard skills of suicide risk assessment.

${ }_{1}^{1}$ Department of Psychological Medicine, Changi General Hospital, Singapore

Correspondence: Dr Tan Rui Qi, Consultant, Department of Psychological Medicine, Changi General Hospital, 2 Simei Street 3 , Singapore 529889. tan.rui.qi@singhealth.com.sg 
Box 1. Risk factors for suicide in elderly individuals:

Modifiable

- Recent negative life event (e.g. family discord, losses)*

- Social isolation/living alone

- Low socioeconomic status*

- Financial or legal difficulties

- Chronic pain

- Chronic medical illness

- Functional impairment

- Alcohol/substance use

- Access to lethal means

Non-modifiable

- Male gender

- Older age

- History of suicidal attempt(s)

- History of psychiatric illness(s)

- Family history of suicide

* While these risk factors are broadly categorised as modifiable, there

are exceptions in several circumstances. An example of a recent negative life event that is non-modifiable is the death of a spouse.

Although low socioeconomic status is potentially modifiable in the aggregate by the government, it is not modifiable by the affected

individual or the treating healthcare professional.

\section{Soft skills}

Given the stigma surrounding suicide and the possible associated feelings of guilt or shame, patients may find it difficult to disclose such sensitive information. Creating a conducive environment can help to allay some of the anxiety that patients face. An ideal setting would be a quiet enclosed area with minimal external disruptions. The interview should be conducted in the patient's preferred language or dialect as much as possible. Nonverbal skills, such as active listening, providing good eye contact, appearing genuine and maintaining a non-judgemental stance, should be embodied by the attending primary care physician. Collectively, these elements help to create a safe and therapeutic space for patients to share their concerns.

The interview should begin with open-ended questions, which help to ease patients into talking freely about their issues. Close-ended questions are better employed in the later part of the interview. They can help the interviewer gain more clarity on the earlier responses of the patient. Leading questions should be avoided as they may invite inaccurate responses. An example of a leading question is: "You do not have any suicidal thoughts, right?" The patient may assume that the physician is not expecting any expression of suicidal ideation and feel discouraged from disclosing such thoughts. A more neutral way to ask the question would be: "Do you have any suicidal thoughts?" while providing the patient sufficient time and space to respond. It is also important to use specific terms such as 'suicide' instead of generic terms like 'self-harm', as patients may have different interpretations of the latter and may provide an inaccurate response.

More subtle suicide warning signs in elderly individuals include non-compliance with medical treatment, refusal to access care during emergencies and little engagement during sessions. ${ }^{(10)}$ It is vital to pick up these subtleties during the consultation and to proceed accordingly with a suicide risk assessment. It is also

\section{Box 2. Eliciting details of a suicidal attempt:}

Before the attempt

- Extent of planning of the act

- Intent of the act

- Perceived lethality of the method

During the attempt

- How, when and where was it done

- Attempts to avoid discovery of the act

- Attempts to seek help after the act

After the attempt

- Any regret in carrying out the act

- Presence of any suicidal thoughts currently after surviving the attempt

important to observe the patient's reaction to the questions asked. For example, when asked about suicidal thoughts, a patient may look away and hesitantly reply: "Not really." This suggests that the patient is ambivalent about disclosing his or her suicidal ideation. In such situations, the physician should probe further tactfully.

\section{Hard skills}

Firstly, the nature and extent (e.g. intensity, frequency) of the suicidal ideation should be established. Next, the presence of a specific plan to end one's life (e.g. how, when, where, method of suicide) should be assessed. It is also important to determine whether any preparations have been made (e.g. giving away possessions, tying up 'loose ends', writing a suicide note, researching on the lethal dose of medications). Preparatory behaviour is indicative of a strong intent of suicide. Lastly, assess the availability of the method that the patient plans on using to commit the act (e.g. accessibility of medications to overdose on, accessibility of a gun to shoot oneself). ${ }^{(11)}$

In the event that the patient has already made a suicide or selfharm attempt, further assessment should involve determining what happened before, during and after the act. This is summarised in Box 2.

In addition, the primary care physician should exclude delirium, examine the patient for signs and symptoms of any comorbid psychiatric illness, including cognitive impairment, and explore additional risk factors for suicide and protective factors against suicide.

\section{Exclude delirium}

Patients in a delirious state may develop suicidal ideation. Early identification of delirium can facilitate prompt evaluation and treatment of underlying reversible organic causes.

\section{Examine for signs and symptoms of comorbid psychiatric illness}

The presence of an underlying psychiatric illness influences the suicide risk, care plans and disposition of the patient. For example, compared with a patient without depression, one with depression may have a higher suicide risk, thereby requiring more urgent intervention. It is important to note that depressive symptoms in elderly individuals may present differently from those in younger populations. Instead of complaints of sadness, 


\begin{tabular}{|c|c|c|c|}
\hline \multicolumn{2}{|c|}{ Question } & \multicolumn{2}{|l|}{ Past month } \\
\hline & $\begin{array}{l}\text { Have you wished you were dead or wished you could go to sleep and } \\
\text { not wake up? }\end{array}$ & & \\
\hline 2. & Have you actually had any thoughts about killing yourself? & & \\
\hline \multicolumn{4}{|c|}{$\begin{array}{l}\text { If YES to } 2 \text {, answer Questions } 3,4,5 \& 6 \\
\text { If NO to } 2 \text {, go directly to Question } 6\end{array}$} \\
\hline 3. & Have you thought about how you might do this? & & \\
\hline 4. & $\begin{array}{l}\text { Have you had any intention of acting on these thoughts of killing } \\
\text { yourself, as opposed to you have the thought but definitely would not act } \\
\text { on them? }\end{array}$ & High risk & \\
\hline 5. & $\begin{array}{l}\text { Have you started to work out or worked out the details of how to kill } \\
\text { yourself? Do you intend to carry out this plan? }\end{array}$ & High risk & \\
\hline \multicolumn{2}{|r|}{ (Always ask Question 6) } & Lifetime & Past 3 months \\
\hline 6. & $\begin{array}{l}\text { Have you done anything, started to do anything, or prepared to do } \\
\text { anything to end your life? } \\
\text { Example: collected pills, obtained a gun, gave away valuables, wrote a } \\
\text { will or suicide note, held a gun but changed your mind, cut yourself, tried } \\
\text { to hang yourself, etc }\end{array}$ & & High risk \\
\hline
\end{tabular}

Fig. 1 Chart shows the Columbia Suicide Severity Rating Scale (reproduced with compliments of The Columbia Lighthouse Project, http://cssrs.columbia.edu/)

elderly individuals may present with poorer memory - hence the term 'pseudodementia' - and excessive preoccupation. ${ }^{(12)}$ Other symptoms include insomnia, appetite changes, loss of interest, excessive guilt and feelings of hopelessness.

An elderly person with cognitive deficits may have an inaccurate appraisal of his or her life situation and exhibit impaired judgement. Depression and anxiety are also common forms of behavioural and psychological symptoms of dementia. These factors can affect suicide risk and should be considered carefully.

\section{Explore additional risk factors of suicide and protective factors against suicide}

A good understanding of these factors helps to guide appropriate interventions to mitigate suicide risk. This is achieved by reducing modifiable risk factors for suicide and reinforcing protective factors against it.

\section{Suicide risk assessment tools}

The Columbia Suicide Severity Rating Scale (C-SSRS) is one of the many tools available to facilitate suicide risk assessment. It is easily administered and has strong evidence in its validity across various cultures, languages and ages. ${ }^{(13,14)}$ There are several versions of the scale for use in different settings, including primary care. There is also a version available for use in patients with cognitive impairment. It comprises only six ' $\mathrm{Yes} / \mathrm{No}^{\prime}$ questions and stratifies patients accordingly into three levels of risk (Fig. 1). The SAD PERSONS scale is another commonly used tool. ${ }^{(15)}$ Unlike C-SSRS, this scale considers interpersonal and environmental factors, which have bearings on the suicide risk of the patient. It also quantifies the severity of suicide risk into three levels. There is no single tool that exists as a gold standard. A proper suicide risk assessment should not be based solely on a cut-off score of a tool but is best undertaken using a holistic approach, involving history-taking, mental state examination and a healthy dose of clinical judgement.

\section{MANAGEMENT OF SUICIDAL PATIENTS}

Immediate psychiatric care is paramount for patients who are highly suicidal. They should be sent to a psychiatric institution, such as the Institute of Mental Health (IMH) in our local context, for an urgent assessment. They will likely require inpatient admission for stabilisation and treatment of any underlying psychiatric illness. However, patients may not always consent to the transfer. This highlights the relevance of Section 9 of the Mental Health (Care and Treatment) Act in primary care settings. It states, "where a medical practitioner has under his care a person believed to be mentally disordered or to require psychiatric treatment, he may send the person to a designated medical practitioner at a psychiatric institution for treatment".(16) Upon transfer, the designated medical practitioner at IMH may admit these patients, even if it is against their will, under Section 10 of the Act. Detailed and clear documentation of the risk assessment should be conducted by the referring primary care physician in order to facilitate communication between healthcare providers and to ensure continuity of care.

However, the management of cases that are less clear-cut can be challenging. An example of such a patient would be an elderly widowed woman who is passively suicidal, has a history of a suicidal attempt and has been experiencing chronic pain. In these instances, it is important to determine the patient's insight regarding his or her condition and receptiveness to receiving treatment. These factors are reflective of one's willingness to seek help in the event of a crisis. Suicide risk can be further mitigated by eliminating as many modifiable suicide risk factors as possible. Examples include treatment of any underlying psychiatric illness, restricting accessibility to methods and reducing identifiable stressors. Establishing the strength of the patient's psychosocial support system is also necessary, as it has bearings on the practicalities of implementing safety measures. These measures include being closely supervised, limiting accessibility to lethal weapons, securing of window grills, safeguarding of medications or toxic agents and being aware of resources available in the event of a crisis (e.g. crisis hotlines, emergency psychiatric services).

If the patient in the example given above demonstrates good insight and receptiveness to treatment and has a decent support system and reasonable safety plans in place, an outpatient referral to a psychiatrist is appropriate. Given the dynamic nature of suicidality, it is recommended that the attending physician continues to conduct regular clinical reviews with ongoing suicide risk assessments of the patient while awaiting a specialist's 
opinion. Ultimately, the physician may still opt to send the patient for an urgent psychiatric assessment if the risk escalates.

A challenge that may arise is the issue of confidentiality. Patients may not consent to physicians disclosing their suicide risk to their family. The physician should carefully consider and weigh the duty to maintain confidentiality with the duty of acting in the best interest of the patient. In situations where concerns about the patient's safety override concerns of confidentiality, a breach of confidentiality can be justified. The physician is then obligated to inform the patient's family or relevant individuals about the patient's suicide risk.

\section{COMMUNITY RESOURCES}

Many community resources are available to support suicidal patients. They include family service centres, Samaritans of Singapore and Silver Ribbon. There are also community mental health teams that provide outreach services to elderly individuals. Examples include the Community Psychogeriatric Programme at Changi General Hospital, Aged Psychiatry Community Assessment and Treatment Service at $\mathrm{IMH}$, and Geriatrics Psychiatry Out-Reach Assessment, Consultation and Enablement Service at the National University Hospital.

\section{CONCLUSION}

Primary care physicians form the bedrock of our healthcare system and play a crucial role in the detection and assessment of suicide risk. With the right blend of soft and hard skills, a proper and thorough suicide risk assessment conducted in a timely manner will help guide management strategies and allocate patients to the most appropriate care setting.

\section{REFERENCES}

1. Chia BH, Chia A. Suicide in Singapore. Singapore Fam Physician 2010; 36:26-9.

2. Rashith R. Number of suicides among seniors hits record high. The Straits Times 2018 Jul 30. Available at: https://www.straitstimes.com/singapore/number-ofsuicides-among-seniors-hits-record-high. Accessed March 6, 2020.

3. Boffin N, Van Casteren V, De Ridder K. Care of general practice patients preceding and following a suicide attempt: observational study in Flemish general practices. BMJ Open 2019; 9:e028546.

4. van Wijngaarden E, Leget C, Goossensen A. Ready to give up on life: the lived experience of elderly people who feel life is completed and no longer worth living. Soc Sci Med 2015; 138:257-64.

5. Raue PJ, Ghesquiere AR, Bruce ML. Suicide risk in primary care: identification and management in older adults. Curr Psychiatry Rep 2014; 16:466.

6. Segal DL. Levels of knowledge about suicide facts and myths among younger and older adults. Clin Gerontol 2000; 22:71-80.

7. Tang NK, Crane C. Suicidality in chronic pain: a review of the prevalence, risk factors and psychological links. Psychol Med 2006; 36:575-86.

8. Fässberg MM, Cheung G, Canetto SS, et al. A systematic review of physical illness, functional disability, and suicidal behaviour among older adults. Aging Ment Health 2016; 20:166-94.

9. World Health Organization. Preventing suicide: a global imperative. Available at: https://www.who.int/publications-detail/preventing-suicide-a-globalimperative. Accessed March 10, 2020

10. Rudd MD. Suicide warning signs in clinical practice. Curr Psychiatry Rep 2008; 10:87-90.

11. Suicide Prevention Resource Center. Suicide assessment and prevention for older adults. Available at: https://www.sprc.org/resources-programs/suicideassessment-prevention-older-adults. Accessed March 3, 2020.

12. Peh LH, Loh C, Chan HN. Consultations in Geriatric Psychiatry: Depression. Singapore: Hope Story Publisher, 2007: 65-7.

13. Posner K, Brown GK, Stanley B, et al. The Columbia-Suicide Severity Rating Scale: initial validity and internal consistency findings from three multisite studies with adolescents and adults. Am J Psychiatry 2011; 168:1266-77.

14. Abarca C, Gheza C, Coda C, Elicer B. Literature review to identify standardized scales for assessing adult suicide risk in the primary health care setting. Medwave 2018; 18:e7246.

15. Patterson WM, Dohn HH, Bird J, Patterson GA. Evaluation of suicidal patients: the SAD PERSONS scale. Psychosomatics 1983; 24:343-5, 348-9.

16 Singapore Statutes Online. Mental Health (Care and Treatment) Act (Chapter 178A). Available at: https://sso.agc.gov.sg/Act/MHCTA2008. Accessed March 9, 2020. 